\title{
A Dichotomy for Local Small-Bias Generators
}

\author{
Benny Applebaum ${ }^{1, \star}$, Andrej Bogdanov ${ }^{2, \star \star}$, and Alon Rosen ${ }^{3, \star \star \star}$ \\ 1 School of Electrical Engineering, Tel-Aviv University \\ benny. applebaum@gmail.com \\ 2 Department of Computer Science and Engineering and Institute for Theoretical \\ Computer Science and Communications, Chinese University of Hong Kong \\ andrejb@cse. cuhk.edu.hk. \\ 3 Efi Arazi School of Computer Science, IDC Herzliya \\ alon.rosen@idc.ac.il
}

\begin{abstract}
We consider pseudorandom generators in which each output bit depends on a constant number of input bits. Such generators have appealingly simple structure: they can be described by a sparse inputoutput dependency graph $G$ and a small predicate $P$ that is applied at each output. Following the works of Cryan and Miltersen (MFCS '01) and by Mossel et al (FOCS '03), we ask: which graphs and predicates yield "small-bias" generators (that fool linear distinguishers)?

We identify an explicit class of degenerate predicates and prove the following. For most graphs, all non-degenerate predicates yield small-bias generators, $f:\{0,1\}^{n} \rightarrow\{0,1\}^{m}$, with output length $m=n^{1+\epsilon}$ for some constant $\epsilon>0$. Conversely, we show that for most graphs, degenerate predicates are not secure against linear distinguishers, even when the output length is linear $m=n+\Omega(n)$. Taken together, these results expose a dichotomy: every predicate is either very hard or very easy, in the sense that it either yields a small-bias generator for almost all graphs or fails to do so for almost all graphs.

As a secondary contribution, we give evidence in support of the view that small bias is a good measure of pseudorandomness for local functions with large stretch. We do so by demonstrating that resilience to linear distinguishers implies resilience to a larger class of attacks for such functions.
\end{abstract}

Keywords: small-bias generator, dichotomy, local functions, NC0.

\section{Introduction}

In recent years there has been interest in the study of cryptographic primitives that are implemented by local functions, that is functions in which each output bit depends on a constant number of input bits. This study has been in large

\footnotetext{
* Supported by Alon Fellowship, by the Israel Science Foundation (grant No. 1155/11), and by the Check Point Institute for Information Security.

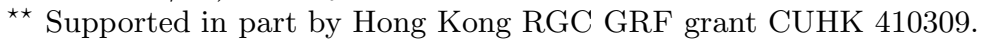

$\star \star \star$ Supported by the Israel Science Foundation (grant No. 334/08).
} 
part spurred by the discovery that, under widely accepted cryptographic assumptions, local functions can achieve rich forms of cryptographic functionality, ranging from one-wayness and pseudorandom generation to semantic security and existential unforgeability [6].

Local functions have simple structure: they can be described by a sparse input-output dependency graph and a sequence of small predicates applied at each output. Besides allowing efficient parallel evaluation, this simple structure makes local functions amenable to analysis, and gives hope for understanding their computational properties. Given that the cryptographic functionalities that local functions can achieve are quite complex, it is very interesting and appealing to try to understand which properties of local functions (namely, graphs and predicates) are necessary and sufficient for them to implement such functionalities.

In this work we focus on the study of local pseudorandom generators with large stretch. We give evidence that for most graphs, all but a handful of "degenerate" predicate types yield pseudorandom generators with output length $m=n^{1+\varepsilon}$ for some constant $\varepsilon>0$. Conversely, we show that for almost all graphs, degenerate predicates are not secure even against linear distinguishers. Taken together, these results expose a dichotomy: every predicate is either very hard or very easy, in the sense that it either yields a small-bias generator for almost all graphs or fails to do so for almost all graphs.

\subsection{Easy, Sometimes Hard, and Almost Always Hard Predicates}

Recall that a pseudorandom generator is a length-increasing function $f:\{0,1\}^{n} \rightarrow$ $\{0,1\}^{m}$ such that no efficiently computable test can distinguish with noticeable advantage between the value $f(x)$ and a randomly chosen $y \in\{0,1\}^{m}$, when $x \in\{0,1\}^{n}$ is chosen at random. The additive stretch of $f$ is defined to be the difference between its output length $m$ and its input length $n$.

In the context of constructing local pseudorandom generators of superlinear stretch, we may assume without loss of generality that all outputs apply the same predicate $P:\{0,1\}^{d} \rightarrow\{0,1\}$ We are interested in understanding which $d$-local functions $f_{G, P}:\{0,1\}^{n} \rightarrow\{0,1\}^{m}$, described by a graph $G$ and a predicate $P$, are pseudorandom generators. For a predicate $P$, we will say

- $P$ is easy if $f_{G, P}$ is not pseudorandom for every $G$ (against a given class of adversaries),

- $P$ is sometimes hard if $f_{G, P}$ is pseudorandom for some $G$, and

- $P$ is almost always hard if $f_{G, P}$ is pseudorandom for a $1-o(1)$ fraction of graphs $G 2$

${ }^{1}$ If this is not the case, project on the outputs labeled by the most frequent predicate. This decreases the stretch only by a constant factor as there are only $2^{2^{d}}$ different predicates.

2 One cannot hope for always hard predicates, for which $f_{G, P}$ is pseudorandom for all graphs, as there are simple examples of "easy" graphs $G$ for which $f_{G, P}$ fails to be pseudorandom regardless of $P$. 
Cryan and Miltersen [17] and Mossel et al. 27] identified several classes of predicates that are easy for polynomial time algorithms when the stretch is a sufficiently large linear function. These include four types of predicates:

1. linear predicates, i.e., $P(w)=b+\Sigma_{i} w_{i}(\bmod 2)$ where $b \in\{0,1\}$,

2. unbalanced predicates, i.e., $\operatorname{Pr}_{w}[P(w)=1] \neq \frac{1}{2}$,

3. predicates that are biased towards one input, i.e., $\operatorname{Pr}_{w}\left[P(w)=w_{i}\right] \neq \frac{1}{2}$,

4. predicates that are biased towards a pair of inputs, i.e., $\operatorname{Pr}_{w}\left[P(w)=w_{i}+w_{j}\right.$ $(\bmod 2)] \neq \frac{1}{2}$.

We call such predicates degenerate. It turns out that all predicates of locality at most 4 are degenerate.

On the positive side, Mossel et al. [27] also gave examples of 5-bit predicates that are sometimes (exponentially) hard against linear distinguishers. Applebaum et al. 5] show that when the locality is sufficiently large, almost always hard predicates against linear distinguishers exist.

Pseudorandomness against linear distinguishers means that there is no subset of output bits whose XOR has noticeable bias. This notion, due to Naor and Naor [28, was advocated in the context of local pseudorandom generators by Cryan and Miltersen [17]. A bit more formally, for a function $f:\{0,1\}^{n} \rightarrow$ $\{0,1\}^{m}$, we let

$$
\operatorname{bias}(f)=\max _{L}\left|\operatorname{Pr}\left[L\left(f\left(\mathcal{U}_{n}\right)\right)=1\right]-\operatorname{Pr}\left[L\left(\mathcal{U}_{m}\right)=1\right]\right|,
$$

where the maximum is taken over all affine functions $L: \mathbb{F}_{2}^{m} \rightarrow \mathbb{F}_{2}$. A small-bias generator is a function $f$ for which bias $(f)$ is small (preferrably negligible) as a function of $n$.

\subsection{Our Results}

We fully classify predicates by showing that all predicates that are not known to be easy, are almost always hard.

Theorem 1 (Non-degenerate predicates are hard). Let $P:\{0,1\}^{d} \rightarrow$ $\{0,1\}$ be any non-degenerate predicate. Then, for every $\varepsilon<1 / 4$ and $m=n^{1+\varepsilon}$ :

$$
\operatorname{Pr}_{G}\left[\operatorname{bias}\left(f_{G, P}\right) \leq \delta(n)\right]>1-o(1)
$$

where $\delta(n)=\exp \left(-\Omega\left(n^{1 / 4-\varepsilon}\right)\right)$ and $G$ is randomly chosen from all d-regular hypergraphs with $n$ nodes (representing the inputs) and $m$ hyperedges (representing the outputs).

The theorem shows that, even when locality is large, the only easy predicates are degenerate ones, and there are no other "sources of easiness" other than ones that already appear in predicates of locality 4 or less.

Conversely, we show that degenerate predicates are easy for linear distinguishers (as opposed to general polynomial-time distinguishers). 
Theorem 2 (Linear tests break degenerate predicates). For every $m=$ $n+\Omega(n)$, and every degenerate predicate $P:\{0,1\}^{d} \rightarrow\{0,1\}$

$$
\underset{G}{\operatorname{Pr}}\left[\operatorname{bias}\left(f_{G, P}\right)>\Omega(1 / \log (n))\right]>1-o(1),
$$

where $G$ is randomly chosen from all $d$-regular hypergraphs with $n$ nodes and $m$ hyperedges.

The proof of Theorem 2 mainly deals with degenerate predicates that are correlated with a pair of their inputs; In this case, we show that the non-linear distinguisher which was previously used in 27] and was based on a semi-definite program for MAX-2-LIN 21] can be replaced with a simple linear distinguisher. (The proof for other degenerate predicates follows from previous works).

Taken together, Theorems 1 and 2 expose a dichotomy: a predicate can be either easy (fail for almost all graphs) or hard (succeeds for almost all graphs). One possible interpretation of our results is that, from a designer point of view, a strong emphasis should be put on the choice of the predicate, while the choice of the input-output dependency graph may be less crucial (since if the predicate is appropriately chosen then most graphs yield a small-bias generator). In some sense, this means that constructions of local pseudorandom generators with large stretch are robust: as long as the graph $G$ is "typical," any non-degenerate predicate can be used (our proof classifies explicitly what is a typical family of graphs and in addition shows that even a mixture of different non-degenerate predicates would work).

\subsection{Why Polynomial Stretch?}

While Applebaum et al. 6] give strong evidence that local pseudorandom generators exist, the stretch their construction achieves is only sublinear, that is $m=n+n^{1-\varepsilon}$. (This stretch can be achieved even for 4-local predicates which are necessarily degenerate.) In contrast, the regime of large (polynomial or even linear) stretch is not as well understood, and the only known constructions are based on non-standard assumptions. (See Section 1.5.)

Local generators of large stretch have several applications in cryptography and complexity, such as secure computation with constant overhead 24] and strong (average-case) inapproximability results for constraint-satisfaction problems [7]. These results are not known to follow from other (natural) assumptions. It should be mentioned that it is possible to convert small polynomial stretch of $m=$ $n^{1+\varepsilon}$ into arbitrary (fixed) polynomial stretch of $m=n^{c}$ at the expense of constant blow-up in the locality. (This follows from standard techniques, see [4] for details). Hence, it suffices to focus on the case of $m=n^{1+\varepsilon}$ for some fixed $\varepsilon$.

The proof of Theorem 1 yields exponentially small bias when $m=O(n)$, and sub-exponential bias for $m=n^{1+\varepsilon}$ where $\varepsilon<1 / 4$. We do not know whether this is tight, but it can be shown that some non-degenerate predicates become easy (to break on a random graph) when the output length is $m=n^{2}$ or even $m=$ $n^{3 / 2}$. In general, it seems that when $m$ grows the number of hard predicates of 
locality $d$ decreases, till the point $m^{\star}$ where all predicates become easy. (By [27, $m^{\star} \leq n^{d / 2}$.) It will be interesting to obtain a classification for larger output lengths, and to find out whether a similar dichotomy happens there as well.

\subsection{Why Small-Bias?}

Small-bias generators are a strict relaxation of cryptographic pseudorandom generators in that the tests $L: \mathbb{F}_{2}^{m} \rightarrow \mathbb{F}_{2}$ are restricted to be affine (as opposed to arbitrary efficiently computable functions). Even though affine functions are, in general, fairly weak distinguishers, handling them is a necessary first step towards achieving cryptographic pseudorandomness. In particular, affine functions are used extensively in cryptanalysis and security against them already rules out an extensive class of attacks.

For local pseudorandom generators with linear stretch, Cryan and Miltersen conjectured that affine distinguishers are as powerful as polynomial-time distinguishers [17. In Section [5, we attempt to support this view by showing that resilience against small-bias, by itself, leads to robustness against other classes of attacks.

Small-bias generators are also motivated by their own right being used as building blocks in constructions that give stronger forms of pseudorandomness. This includes constructions of local cryptographic pseudorandom generators [7/4], as well as pseudorandom generators that fool low-degree polynomials [14, small-space computations [23], and read-once formulas [1].

\subsection{Related Work}

The function $f_{G, P}$ was introduced by Goldreich [22] who conjectured that when $m=n$, one-wayness should hold for a random graph and a random predicate. This view is supported by the results of [22 29 3|16 26 20|25] who show that a large class of algorithms (including ones that capture DPLL-based heuristics) fail to invert $f_{G, P}$ in polynomial-time.

At the linear regime, i.e., when $m=n+\Omega(n)$, it is shown in [12] that if the predicate is degenerate the function $f_{G, P}$ can be inverted in polynomial-time. (This strengthens the results of 17/27] who only give distinguishers.) Recently, a strong self-amplification theorem was proved in [13] showing that for $m=$ $n+\Omega_{d}(n)$ if $f_{G, P}$ is hard-to-invert over tiny (sub-exponential small) fraction of the inputs with respect to sub-exponential time algorithm, then the same function is actually hard-to-invert over almost all inputs (with respect to subexponential time algorithms).

Pseudorandom generators with sub-linear stretch can be implemented by 4local functions based on standard intractability assumptions (e.g., hardness of factoring, discrete-log, or lattice problems) [6], or even by 3-local functions based on the intractability of decoding random linear codes 8 . However, it is unknown how to extend this result to polynomial or even linear stretch since all known stretch amplification procedures introduce a large (polynomial) overhead in the 
locality. In fact, for the special case of 4-local functions (in which each output depends on at most 4 input bits), there is a provable separation: Although such functions can compute sub-linear pseudorandom generators [6] they cannot achieve polynomial-stretch [1727.

Alekhnovich [1] conjectured that for $m=n+\Theta(n)$, the function $f_{G, P}$ is pseudorandom for a random graph and when $P$ is a randomized predicate which computes $z_{1} \oplus z_{2} \oplus z_{3}$ and with some small probability $p<\frac{1}{2}$ flips the result. Although this construction does not lead directly to a local function (due to the use of noise), it was shown in [7] that it can be derandomized and transformed into a local construction with linear stretch. (The restriction to linear stretch holds even if one strengthen Alekhnovich's assumption to $m=\operatorname{poly}(n)$.)

More recently, 4] showed that the pseudorandomness of $f_{G, P}$ with respect to a random graph and output length $m$, can be reduced to the one-wayness of $f_{H, P}$ with respect to a random graph $H$ and related output length $m^{\prime}$ (for certain settings of the stretch and security parameters). The current paper complements this result as it provides a criteria for choosing the predicate $P$.

\section{Techniques and Ideas}

In this section we give an overview of the proof of Theorem 1 Let $f:\{0,1\}^{n} \rightarrow$ $\{0,1\}^{m}$ be a $d$-local function where each output bit is computed by applying some $d$-local predicate $P:\{0,1\}^{d} \rightarrow\{0,1\}$ to a (ordered) subset of the inputs $S \subseteq[n]$. Any such function can be described by a list of $m d$-tuples $G=\left(S_{1}, \ldots, S_{m}\right)$ and the predicate $P$. Under this convention, we let $f_{G, P}:\{0,1\}^{n} \rightarrow\{0,1\}^{m}$ denote the corresponding $d$-local function.

We view $G$ as a $d$-regular hypergraph with $n$ nodes (representing inputs) and $m$ hyperedges (representing outputs) each of size $d$. (We refer to such a graph as an $(m, n, d)$-graph.) Since we are mostly interested in polynomial stretch we think of $m$ as $n^{1+\varepsilon}$ for some fixed $\varepsilon>0$, e.g., $\varepsilon=0.1$.

We would like to show that for almost all $(m, n, d)$-graphs $G$, the function $f_{G, P}$ fools all linear tests $L$, where $P$ is non-degenerate. Following [27, we distinguish between light linear tests which depend on less than $k=\Omega\left(n^{1-2 \varepsilon}\right)$ outputs, and heavy tests which depend on more than $k$ outputs.

From our definition of non-degenerate predicates, it immediately follows that such predicates $P$ satisfy two forms of "non-linearity": (1) (2-resilience) $P$ is uncorrelated with any linear function in two or fewer inputs; and (2) (algebraic nonlinearity) $P$ is not linear as a polynomial over $\mathbb{F}_{2}$. Both properties are classical design criteria which are widely used in practical cryptanalysis (cf. [30]). We use the fist property to fool light linear tests (tests that depend on a small number of outputs) and the second one to fool heavy linear tests (tests that depend on a large number of outputs).

\subsection{Fooling Light Tests}

Our starting point is a result of [27] which shows that if the predicate is the parity predicate $\oplus$ and the graph is a good expander, the output of $f_{G, \oplus}\left(\mathcal{U}_{n}\right)$ 
perfectly fools all light linear tests. In terms of expectation, this can be written as

$$
\underset{x}{\mathrm{E}}\left[L\left(f_{G, \oplus}(x)\right)=0\right],
$$

where we think of $\{0,1\}$ as $\{ \pm 1\}$, and let $L:\{ \pm 1\}^{m} \rightarrow\{ \pm 1\}$ be a light linear test. Our key insight is that the case of a general predicate $P$ can be reduced to the case of linear predicates.

More precisely, let $\xi$ denote the outcome of the test $L\left(f_{G, P}(x)\right)$. Then, by looking at the Fourier expansion of the predicate $P$, we can write $\xi$ as a convex combination over the reals of exponentially many summands of the form $\xi_{i}=L\left(f_{G_{i}, \oplus}(x)\right)$ where the $G_{i}$ 's are subgraphs of $G$ in the sense that the $j$-th hyperedge of $G_{i}$ is a subset of the $j$-th hyperedge of $G$. (The exact structure of $G_{i}$ is determined by the Fourier representation of $P$.) When $x$ is uniformly chosen, the random variable $\xi$ is a weighted sum (over the reals) of many dependent random variables $\xi_{i}$ 's. However, if all the subgraphs are good expanders, the expectation of each summand $\xi_{i}$ is zero, and so, by the linearity of expectation, the expectation of $\xi$ is also zero.

It turns out that when the predicate is 2-resilient the size of each hyperedge of $G_{i}$ is at least 3 , and therefore if every 3-uniform subgraph of $G$ is a good expander $f_{G, P}$ (perfectly) passes all light linear tests. Fortunately, it turns out that most graphs $G$ satisfy this property. We emphasize that the argument crucially relies on the perfect bias of XOR predicates, as there are exponentially many summands. (See Section 3.1 for full details.)

\subsection{Fooling Heavy Tests}

Consider a heavy test which involves $t \geq k$ outputs. Switching back to zero-one notation, assume that the test outputs the value $\xi=P\left(x_{S_{1}}\right)+\ldots+P\left(x_{S_{t}}\right)$ $(\bmod 2)$ where $x \stackrel{R}{\leftarrow} \mathcal{U}_{n}$. Our goal is to show that $\xi$ is close to a fair coin. For this it suffices to show that the sum $\xi$ can be rewritten as the sum (over $\mathbb{F}_{2}$ ) of $\ell$ random variables

$$
\xi=\xi_{1}+\ldots+\xi_{\ell} \quad(\bmod 2),
$$

where each random variable $\xi_{i}$ is an independent non-constant coin, i.e., $\operatorname{Pr}\left[\xi_{i}=\right.$ $1] \in\left[2^{-d}, 1-2^{-d}\right]$. In this case, the statistical distance between $\xi$ and a fair coin is exponentially small (in $\ell$ ), and we are done as long as $\ell$ is large enough.

In order to partition $\xi$, let us look at the hyperedges $S_{1}, \ldots, S_{t}$ which are involved in the test. As a first attempt, let us collect $\ell$ distinct "independent" hyperedges that do not share a single common variable. Renaming the edges, we can write $\xi$ as

$$
\left(P\left(x_{T_{1}}\right)+\ldots+P\left(x_{T_{\ell}}\right)\right)+\left(P\left(x_{S_{\ell+1}}\right)+\ldots+P\left(x_{S_{t}}\right)\right) \quad(\bmod 2),
$$

where the first $\ell$ random variables are indeed statistically independent. However, the last $t-\ell$ hyperedges violate statistical-independence as they may be correlated with more than one of the first $\ell$ hyperdges. This is the case, for example, if $S_{j}$ has a non-empty intersection with both $T_{i}$ and $T_{r}$. 
This problem is fixed by collecting $\ell$ "strongly-independent" hyperedges $T_{1}$, $\ldots, T_{\ell}$ for which every $S_{j}$ intersects at most a single $T_{i}$. (Such a large collection is likely to exist since $t$ is sufficiently large.) In this case, for any fixing of the variables outside the $T_{i}$ 's, the random variable $\xi$ can be partitioned into $\ell$ independent random variables of the form $\xi_{i}=P\left(x_{T_{i}}\right)+\sum P\left(x_{S_{j}}\right)$, where the sum ranges over the $S_{j}$ 's which intersects $T_{i}$. This property (which is a relaxation of Eq. 1) still suffices to achieve our goal, as long as the $\xi_{i}$ 's are non-constant.

To prove the latter, we rely on the fact that $P$ has algebraic degree 2 . Specifically, let us assume that $S_{i}$ and $T_{j}$ have no more than a single common input node. (This condition can be typically met at the expense of throwing a small number of the $T_{i}$ 's.) In this case, the random variable $\xi_{i}=P\left(x_{T_{i}}\right)+\sum P\left(x_{S_{j}}\right)$ cannot be constant, as the first summand is a degree 2 polynomial in $x_{T_{i}}$ and each of the last summands contain at most a single variable from $T_{i}$. Hence, $\xi_{i}$ is a non-trivial polynomial whose degree is lower-bounded by 2 . This completes the argument. Interestingly, non-linearity is used only to prove that the $\xi_{i}$ 's are non-constant. Indeed, linear predicates fail exactly for large tests for which the $\xi_{i}$ 's become fixed due to local cancelations. (See Section 3.2 for details.)

\subsection{Proving Theorem 2}

When $P$ is a degenerate predicate and $G$ is random, the existence of a linear distinguisher follows by standard arguments. The cases of linear or biased $P$ are trivial, and the case of bias towards one input was analyzed by Cryan and Miltersen. When $P$ is biased towards a pair of inputs, say the first two, we think of $P$ as an "approximation" of the parity $x_{1} \oplus x_{2}$ of its first two inputs. If $P$ happened to be the predicate $x_{1} \oplus x_{2}$, one could find a short "cycle" of output bits that, when XORed together, causes the corresponding input bits to cancel out. In general, as long as the outputs along the cycle do not share any additional input bits, the output of the test will be biased, with bias exponential in the length of the cycle. In Section 4 we show that a random $G$ is likely to have such short cycles, and so the corresponding linear test will be biased.

\section{Non-degenerate Predicates Are Hard}

In this section we prove Theorem 1. We follow the outline described in Section 2 and handle light linear tests and heavy linear tests separately.

\subsection{Fooling Light Tests}

In this section we show that if the predicate $P$ is 2 -resilient (see definition below) and the graph $G$ is a good expander, the function $f_{G, P}$ is $k$-wise independent, and in particular fools linear tests of weight smaller than $k$. We will need the following definitions. 
Super expansion. Let $G$ be an $(m, n, d)$-graph. A graph $H$ is $(k, a)$ subgraph of $G$ if it can be constructed by choosing $\ell \leq k$ distinct hyperedges of $G$ and for each selected hyperedge $S_{j}$ removing some of the nodes while leaving $b_{j} \geq a$ nodes. We say that $G$ is $(k, a)$ super-expander if the hyperedges $T=T_{1}, \ldots, T_{\ell}$ of every $(k, a)$-subgraph $H$ of $G$ touch more than $b \ell / 2$ nodes where $b=\sum\left|T_{j}\right| / \ell$ is the average cardinality of the hyperedges of $H$. We say that $G$ is $(k, a)$-linear if the hyperedges of every $(k, a)$-subgraph of $G$ are linearly independent viewed as vectors in $\mathbb{F}_{2}^{n}$.

Fourier coefficients. For a set $T \subseteq[d]$, let $\chi_{T}:\{ \pm 1\}^{d} \rightarrow\{ \pm 1\}$ be the Parity function defined by $\left(x_{1}, \ldots, x_{d}\right) \mapsto(-1)^{\sum_{t \in T} x_{t}}$. It is well known that every predicate $P:\{ \pm 1\}^{d} \rightarrow\{ \pm 1\}$ can be expressed as a convex combination of parities, i.e., $P(x)=\sum_{T \subseteq[d]} \alpha_{T} \chi_{T}(x)$ where $\alpha_{T} \in \mathbb{R}$. The predicate is a-resilient if $\alpha_{T}$ is zero for every set $T$ of size smaller or equal to $a$.

The following lemma shows that resiliency combined with $(k, a)$-linearity leads to $k$-wise independence.

Lemma 1. If $P$ is $(a-1)$-resilient and the $(m, n, d)$-graph $G$ is $(k, a)$-linear then $f_{G, P}$ is $k$-wise independent generator, i.e., the $m$ r.v.'s $\left(y_{1}, \ldots, y_{m}\right)=f_{G, P}\left(\mathcal{U}_{n}\right)$ are $k$-wise independent.

Proof. Fix an $\ell \leq k$ outputs of $f_{G, P}$, and let $S_{1}, \ldots, S_{\ell}$ be the corresponding hyperedges. We should show that $\mathrm{E}_{x}\left[\prod_{i} P\left(x_{S_{i}}\right)\right]=0$. For every $x \in\{0,1\}^{n}$ we have:

$$
\prod_{i=1}^{\ell} P\left(x_{S_{i}}\right)=\prod_{i=1}^{\ell} \sum_{T \subseteq[d],|T| \geq a} \alpha_{T} \chi_{T}\left(x_{S_{i}}\right)=\sum_{\boldsymbol{T}=\left(T_{1}, \ldots, T_{\ell}\right),\left|T_{i}\right| \geq a} \prod_{i} \alpha_{T_{i}} \chi_{S_{i, T_{i}}}(x),
$$

where $S_{i,\left\{K_{1}, \ldots, K_{b}\right\}}$ denotes the set $\left\{S_{i, K_{1}}, \ldots, S_{i, K_{b}}\right\}$ and $S_{i, j}$ denotes the $j$-th entry of the tuple $S_{i}$. Hence, by the linearity of expectation, it suffices to show that

$$
\mathrm{E}\left[\prod_{i} \chi_{S_{i, T_{i}}}(x)\right]=0
$$

for every $\left(T_{1}, \ldots, T_{\ell}\right)$ where $T_{i} \subseteq[d],\left|T_{i}\right| \geq a$. (Recall that the $\alpha_{T_{i}}$ 's are constants and thus can be ignored.) Observe that $\prod_{i} \chi_{S_{i, T_{i}}}(x)$ is just a parity function, which, by $(k, a)$-linearity, is non-constant. Since every non-constant parity function is balanced (guaranteed to have zero expectation value), the claim follows.

Next, we show that $(k, a)$-linearity is implied by super-expansion, and that a random graph is likely to be super-expanding.

Lemma 2. Let $d \geq 3$ be a constant. Let $\Delta \leq \sqrt{n} / \log n$ and $3 \leq a \leq d$.

1. Every $(\Delta n, n, d)$-graph which is $(k, a)$-super-expander is also $(k, a)$-linear.

2. A random $(\Delta n, n, d)$-graph is whp an $\left(\alpha n / \Delta^{2}, a\right)$-super-expander where $\alpha$ is a constant that depends on $a, d{ }^{3}$

${ }^{3}$ With high probability (whp) means with probability $1-o(1)$ as $n$ gets large. 
Proof. The proof of the first item parallels the standard relation between losslessexpansion and unique/odd-expansion. Let $G$ be a $(k, a)$-super-expander. Observe that if $G$ is not $(k, a)$-linear then there must be $(k, a)$-subgraph $H$ whose edges sum-up to zero (over $\mathbb{F}_{2}^{n}$ ). We argue that $G$ cannot have such a subgraph. Indeed, by counting edges, in each $(k, a)$-subgraph $H$ the average degree of the participating nodes is smaller than 2 , and so there exists at least one node which participates in a single hyperedge. Hence, the sum of the hyperedges (over $\mathbb{F}_{2}^{n}$ ) is non-zero.

To prove the second item, we calculate the probability that a random $(\Delta n, n, d)$ graph fails to be $(k, a)$-super-expander. First we bound the probability that there exists a subgraph $H$ with $\ell$ hyperedges and average degree $b \geq a$ that violates expansion. This probability is bounded by

$$
\begin{aligned}
\left(\begin{array}{c}
\Delta n \\
\ell
\end{array}\right) \cdot 2^{d \ell} \cdot\left(\begin{array}{c}
n \\
b \ell / 2
\end{array}\right) \cdot\left(\frac{b \ell}{2 n}\right)^{b \ell} & <\left(\frac{e \Delta n}{\ell} \cdot 2^{d} \cdot\left(\frac{2 e n}{b \ell}\right)^{b / 2}\left(\frac{b \ell}{2 n}\right)^{b}\right)^{\ell} \\
& =\left(e 2^{d}\left(\frac{b e}{2}\right)^{b / 2} \Delta\left(\frac{\ell}{n}\right)^{b / 2-1}\right)^{\ell} \\
& \leq\left(c_{d, a} \Delta\left(\frac{\ell}{n}\right)^{a / 2-1}\right)^{\ell}
\end{aligned}
$$

where $c_{d, a}$ is a constant which depends on $d$ and $a$, and the second inequality is due to $a \leq b \leq d$. Let us denote the above quantity by $p_{\ell, n, \Delta, a, d}$. By a union-bound $G$ fails to be $(k, a)$-super-expander with probability at most $\sum_{2 \leq \ell \leq k} p_{\ell, n, \Delta, a, d}$.

Let us fix $a \geq 3$, and assume that $\Delta \leq n^{\frac{1}{2}} / \log n$ and $k=\alpha n / \Delta^{2}$ where $\alpha=1 /\left(2 c_{d, a}\right)^{2}$ is a constant. Indeed, in this case

$$
p_{\ell} \leq\left(c_{d, a} \frac{\Delta \sqrt{\ell}}{\sqrt{n}}\right)^{\ell} \leq\left(c_{d, a} \frac{\sqrt{\ell}}{\log n}\right)^{\ell} .
$$

Observe that for $\ell=1,2,3$, the quantity $p_{\ell}$ is $o(1)$, for $4 \leq \ell \leq 10 \log n$ the quantity $p_{\ell} \leq O\left(1 / \log ^{2} n\right)$ and for $10 \log n \leq \ell \leq \alpha n / \Delta^{2}$ the quantity $p_{\ell}$ is at most $O\left(1 / n^{10}\right)$. It follows that each of these three intervals contributes $o(1)$ to the overall failure probability.

By combining the lemmas, we obtain the following corollary.

Corollary 1. If $P$ is 2-resilient and $m=\Delta n$ for constant $\Delta$, then whp over the choice of an $(m, n, d)$-graph $G$, the function $f_{G, P}$ is $k$-wise independent for $k=\Omega(n)$. If $\Delta=n^{\varepsilon}$, the above holds with $k=\Omega\left(n^{1-2 \varepsilon}\right)$.

By taking $\varepsilon<1 / 4,2$-resiliency suffices for $\omega(\sqrt{n})$-wise independence whp. 


\subsection{Fooling Heavy Tests}

In this section we show that if the predicate $P$ is non-linear and the graph $G$ has large sets of "independent" hyperedges, the function $f_{G, P}$ fools linear tests of weight larger than $k$. Formally, we will need the following notion of independence.

$(k, \ell, b)$-independence. Let $\mathcal{S}$ be a collection of $k$ distinct hyperedges. A subset $\mathcal{T} \subseteq \mathcal{S}$ of $\ell$ distinct hyperedges is an $(\ell, b)$-independent set of $\mathcal{S}$ if the following two properties hold: (1) Every pair of hyperedges $\left(T, T^{\prime}\right) \in \mathcal{T}$ are of distance at least 2, namely, for every pair $T_{i} \neq T_{j} \in \mathcal{T}$ and $S \in \mathcal{S}$,

$$
T_{i} \cap S=\emptyset \text { or } T_{j} \cap S=\emptyset ;
$$

and (2) For every $T_{i} \in \mathcal{T}$ and $S \neq T_{i}$ in $\mathcal{S}$ we have

$$
\left|T_{i} \cap S\right|<b .
$$

A graph is $(k, \ell, b)$-independent if every set of hyperedges of size larger than $k$ has an $(\ell, b)$-independent set.

Our key lemma shows that good independence and large algebraic degree guarantee resistance against heavy linear tests.

Lemma 3. If $G$ is $(k, \ell, b)$-independent and $P$ has an algebraic degree of at least $b$, then every linear test of size at least $k$ has bias of at most $\frac{1}{2} e^{-2 \ell / 2^{d}}$.

Proof. Fix some test $\mathcal{S}=\left(S_{1}, \ldots, S_{k}\right)$ of size $k$, and let $\mathcal{T}=\left(T_{1}, \ldots, T_{\ell}\right)$ be an $(\ell, b)$-independence set of $\mathcal{S}$. Fix an arbitrary assignment $\sigma$ for all the input variables which do not participate in any of the $T_{i}$ 's and choose the other variables uniformly at random. In this case, we can partition the output of the test $y$ to $\ell$ summands over $\ell$ disjoint blocks of variables, namely

$$
y=\sum_{i \in[k]} P\left(x_{S_{i}}\right)=\sum_{i \in[\ell]} z_{i}\left(x_{T_{i}}\right),
$$

where the sum is over $\mathbb{F}_{2}$ and

$$
z_{i}\left(x_{T_{i}}\right)=P\left(x_{T_{i}}\right)+\sum_{S: T_{i} \neq S, S \cap T_{i} \neq \emptyset} P\left(x_{S \cap T_{i}}, \sigma_{S \backslash T_{i}}\right) .
$$

We need two observations: (1) the random variables $z_{i}$ 's are statistically independent (as each of them depends on a disjoint block of inputs); and (2) the r.v. $z_{i}$ is non-constant and, in fact, it takes each of the two possible values with probability at least $2^{-d}$. To prove the latter fact it suffices to show that $z_{i}(x)$ is a non-zero polynomial (over $\mathbb{F}_{2}$ ) of degree at most $d$. Indeed, recall that $z_{i}$ is the sum of the polynomial $P\left(x_{T_{i}}\right)$ whose degree is in $[b, d]$, and polynomials of the form $P\left(x_{S \cap T_{i}}, \sigma_{S \backslash T_{i}}\right)$ whose degree is smaller than $b$ (as $\left|S \cap T_{i}\right|<b$ ). Therefore the degree of $z_{i}$ is in $[b, d]$.

To conclude the proof, we note that the parity of $\ell$ independent coins, each with expectation in $(\delta, 1-\delta)$, has bias of at most $\frac{1}{2}(1-2 \delta)^{\ell}$. (See, e.g., [27]). 
We want to show that a random graph is likely to be $(k, \ell, 2)$-independent.

Lemma 4. For every positive $\varepsilon$ and $\delta$. A random $\left(n^{1+\varepsilon}, n, d\right)$-graph is, whp, $\left(n^{2 \varepsilon+\delta}, n^{\delta / 2}, 2\right)$ independent.

Proof. We will need the following claim. Call a hyperedge $S$-intersecting if there exists another hyperedge $S^{\prime}$ in the graph for which $\left|S^{\prime} \cap S\right| \geq b$. We first bound the number of $b$-intersecting hyperedges.

Claim. Let $b$ be a constant. Then, in a random $\left(m=n^{1+\varepsilon}, n, d\right)$-graph, whp, the number of $b$-intersecting hyperedges is at most $n^{2(1+\varepsilon)-b} \log n$.

Hence, whp, at most $O\left(n^{2 \varepsilon} \log n\right)$ of the hyperedges are 2-intersecting, and for $\varepsilon<1 / 4$ there are at most $o(\sqrt{n})$ such hyperedges.

Proof (of Claim). Let $X$ be the random variable which counts the number of $b$-intersecting hyperedges. First, we bound the expectation of $X$ by $m^{2} d^{2 b} / n^{b}=$ $d^{2 b} \cdot n^{2(1+\varepsilon)-b}$. To prove this, it suffices to bound the expected number of pairs $S_{i}, S_{j}$ which $b$-intersects. Each such pair $b$-intersects with probability at most $d^{2 b} / n^{b}$, and so, by linearity of expectation, the expected number of of intersecting pairs is at most $m^{2} d^{2 b} / n^{b}$. Now, by applying Markov's inequality, we have that $\operatorname{Pr}\left[X>\frac{\log n}{d^{2 b}} \mathrm{E}[X]\right]<d^{2 b} / \log n=o(1)$, and the claim follows. (A stronger concentration can be obtained via a martingale argument.)

We can now prove Lemma 4. Assume, without loss of generality, that $\varepsilon>1$ (as if the claim holds for some value of $\varepsilon$ it also holds for smaller values). First observe that, whp, all the input nodes in $G$ have degree at most $2 n^{\varepsilon}$. As by a multiplicative Chernoff bound, the probability that a single node has larger degree is exponentially small in $n^{\varepsilon}$. We condition on this event and the event that there are no more than $r=n^{2 \varepsilon} \log n$ 2-intersecting edges. Fix a set of $k=n^{2 \varepsilon+\delta}$ hyperedges. We extract an $(\ell, 2)$-independent set by throwing away the 2-expanding edges, and then by iteratively inserting an hyperedge $T$ into the independent set and removing all the hyperedges $S$ that share with $T$ a common node, and the hyperedges which share a node with an edge, that shares a node with $T$. At the beginning we removed at most $r$ edges, and in each iteration we remove at most $\left(d 2 n^{\varepsilon}\right)^{2}$ edges, hence there are at least $\ell \geq \frac{k-r}{4 d^{2} n^{2 \varepsilon}}>n^{\delta / 2}$ hyperedges in the independent set.

Combining the lemmas together we get:

Corollary 2. Fix some positive $\varepsilon$ and $\delta$. If $P$ has an algebraic degree of at least 2 and $m=n^{1+\varepsilon}$, then, whp over the choice of a random $(m, n, d)$-graph, the function $f_{G, P}$ has at most sub-exponential bias (i.e., $\exp \left(-\Omega\left(n^{\delta}\right)\right)$ ) against linear tests of size at least $n^{2 \varepsilon+2 \delta}$.

By combining Corollaries 1 and 2. we obtain Theorem 1 .

\section{Linear Tests Break Degenerate Predicates}

In this section we prove Theorem 2, That is, we show that the assumptions that $P$ is non-linear and 2-resilient are necessary for $P$ to be a hard predicate. Clearly the assumption that $P$ is non-linear is necessary even when $m=n+1$. 
When $m \geq K n$ for a sufficiently large constant $K$ (depending on $d$ ), it follows from work of Cryan and Miltersen [17] that if $P$ is not 1-resilient, then for any $f:\{ \pm 1\}^{n} \rightarrow\{ \pm 1\}^{m}$, the output of $f$ is distinguishable from uniform with constant advantage by some linear test. When $P$ is 1-resilient but not 2-resilient, Mossel, Shpilka, and Trevisan show that $f$ is distinguishable from uniform by a polynomial-time algorithm, but not by one that implements a linear test.

Here we show that if $P$ is not 2-resilient, then the output of $f_{G, P}$ is distinguishable by linear tests with non-negligible advantage with high probability over the choice of $G$.

Claim. Assume $P$ is unbiased and 1-resilient but $\left|\mathrm{E}\left[P(z) z_{1} z_{2}\right]\right|=\alpha>0$. Then for every $\ell=o(\log n)$, with probability $1-\left(2^{-\Omega(\ell)}+d \ell / n\right)$ over the choice of $G$, there exists a linear test that distinguishes the output of $f_{G, P}$ from random with advantage $\alpha^{\ell}$.

Proof. Let $H$ be the directed graph with vertices $\{1, \ldots, n\}$ where every hyperedge $\left(i_{1}, i_{2}, \ldots, i_{d}\right)$ in $G$ induces the edge $\left(i_{1}, i_{2}\right)$ in $H$.

Let $\ell$ be the length of the shortest directed cycle in $H$ and without loss of generality assume that this cycle consists of the inputs $1,2, \ldots, \ell$ in that order. Let $z_{i}$ be the name of the output that involves inputs $i$ and $i+1$ for $i$ ranging from 1 to $\ell$ (where $i$ is taken modulo $\ell$ ) and $S_{i}$ the corresponding hyperedge. With probability at least $1-d \ell / n$, input $i$ does not participate in any hyperedge besides $S_{i}$ and $S_{i+1}$ and all other inputs participate in at most one of the hyperedges $S_{1}, \ldots, S_{\ell}$.

We now calculate the bias of the linear test that computes $z_{1} \oplus \ldots \oplus z_{\ell}$. For simplicity, we will assume that $d=3$; larger values of $d$ can be handled analogously but the notation is more cumbersome. We will denote the entries in $S_{i}$ by $i, i+1$ and $i^{\prime}$. Then the fourier expansion of $z_{i}\left(x_{S_{i}}\right)$ has the form

$$
z_{i}\left(x_{S_{i}}\right)=\alpha x_{i} x_{i+1}+\beta x_{i} x_{i^{\prime}}+\gamma x_{i+1} x_{i^{\prime}}+\delta x_{i} x_{i+1} x_{i^{\prime}}
$$

The Fourier expansion of the expression $\mathrm{E}\left[z_{1}\left(x_{S_{1}}\right) \ldots z_{\ell}\left(x_{S_{\ell}}\right)\right]$ can be written as a sum of $4^{\ell}$ products of different monomials participating in the above terms. The only monomial that does not vanish is the one containing all the $\alpha$-terms, namely

$$
\mathrm{E}\left[\prod_{i=1}^{n} \alpha x_{i} x_{i+1}\right]=\alpha^{\ell} .
$$

All the other products of monomials contain at least one unique term of the form $x_{i^{\prime}}$, and this causes the expectation to vanish.

It remains to argue that with high probability $\ell$ is not too large. We show that with probability $1-O\left((4 / K)^{\ell}\right), H$ has a directed cycle of length $\ell$, as long as $\ell<\log _{2 K}(n / 4)$. Let $X$ denote the number of directed cycles of length $\ell$ in $H$. The number of potential directed cycles of length in $H$ is $n(n-1) \ldots(n-\ell+1) \geq$ $(n-\ell)^{\ell}$. Each of these occurs uniquely in $H$ with probability

$$
(K n)(K n-1) \ldots(K n-\ell+1)\left(\frac{1}{n(n-1)}\right)^{\ell}\left(1-\frac{1}{n(n-1)}\right)^{K n-\ell} \geq\left(\frac{K n-\ell}{n^{2}}\right)^{\ell} .
$$


Therefore $\mathrm{E}[X] \geq(K / 4)^{\ell}$. The variance can be upper bounded as follows. The number of pairs of cycles of length $\ell$ that intersect in $i$ edges is at most $\left(\begin{array}{l}\ell \\ i\end{array}\right) n^{2 \ell-i-1}$, and the covariance of the indicators for these cycles is at most $(K / n)^{2 \ell-i}$. Adding all the covariances up as $i$ ranges from 1 to $\ell$, it follows that

$$
\operatorname{Var}[X] \leq \mathrm{E}[X]+\sum_{i=1}^{\ell}\left(\begin{array}{l}
\ell \\
i
\end{array}\right) n^{2 \ell-i-1}\left(\frac{K}{n}\right)^{2 \ell-i} \leq \mathrm{E}[X]+\frac{2^{\ell} K^{2 \ell}}{n} .
$$

By Chebyshev's inequality,

$$
\operatorname{Pr}[X=0] \leq \frac{\operatorname{Var}[X]}{\mathrm{E}[X]^{2}}<\frac{2}{\mathrm{E}[X]}
$$

as long as $\ell<\log _{2 K}(n / 4)$.

\section{Small Bias vs. Cryptographic Security for Local Functions}

It is not difficult to come up with examples of generators that have (exponentially) small bias against linear distinguishers but are not cryptographically secure. However, we do not know of any such examples of generators that are local and have at least linear stretch: To the best of our knowledge, all local functions of linear stretch that are known to implement small-biased generators could be pseudorandom generators against all polynomial-time adversaries.

Therefore it may be plausible to conjecture that if $P$ is almost always hard against linear adversaries, then $P$ is almost always hard against polynomialtime adversaries. While this conjecture cannot be proven without resolving the existence of pseudorandom generators, we give evidence in support of it: We show that if $P$ is almost always hard against linear adversaries, then $f_{G, P}$ is not only small-biased but (1) it is $k$-wise independent and (2) it cannot be inverted by myopic backtracking algorithms.

First, we observe that for local functions the small-bias property immediately implies $k$-wise independence. (This is in general false for non-local functions.)

Lemma 5. Let $f:\{0,1\}^{n} \rightarrow\{0,1\}^{m}$ be a d-local function which is $2^{-k d}$-biased. Then it is also $k$-wise independent.

Proof. Assume towards a contradiction that $f$ is not $k$-wise independent. Then, there exists a set of $k$ outputs $T$ and a linear distinguisher $L$ for which $\varepsilon=$ $\left|\operatorname{Pr}\left[L\left(y_{T}\right)=1\right]-\operatorname{Pr}[L(u)=1]\right|>0$, where $y=f(x)$ for a uniformly random $x$ and $u$ is a uniformly random string of length $k$. Since $f$ is $d$-local, $y_{T}$ is sampled by using fewer than $k d$ bits of randomness and therefore $\varepsilon \geq 2^{-k d}$.

Recall that the proof of our main theorem, Theorem 1, establishes $k$-wise independence as an intermediate step (Section 3.1). However, the above lemma is stronger in the sense that it holds for every fixed graph and every output length including ones that are not covered by the main theorem. 
By plugging in known results about $k$-wise independent distributions, it immediately follows that if a local function is sufficiently small-biased, then it is pseudorandom against $\mathbf{A} \mathbf{C}^{\mathbf{0}}$ circuits [15, linear threshold functions over the reals [18, and degree-2 threshold functions over the reals [19].

Attacks on local functions, which are actively studied at the context of algorithms for constraint-satisfaction problems, appear to be based mainly on "local" heuristics (DPLL, message-passing algorithms, random-walk based algorithms) or linearization [9]. Hence, it appears that in the context of local functions, the small-bias property already covers all "standard" attacks. We support this intuition by showing that if $P$ is non-degenerate, then the outputs of $f_{G, P}$ are not merely min-wise independent, but have a stronger property: Even after reading an arbitrary set of $t$-outputs, the posterior distribution on every set of $\ell$ inputs, while not uniform, still has large min-entropy. We call this property robustness.

The notion of robustness was used by Cook et al. [16] to prove that myopic backtracking algorithms cannot invert $f_{G, P}$ in polynomial time when $m=n$. We now argue that for $f_{G, P}$, robustness is almost always a consequence of small bias, and conclude that $f_{G, P}$ cannot be inverted by myopic backtracking algorithms even when $m=n^{1+\varepsilon}, \varepsilon<1 / 4$, as long as $P$ is non-degenerate. (The analysis of [16] also applies to some degenerate predicates.)

\subsection{Robustness and Myopic Backtracking Algorithms}

Robustness. Let $f:\{0,1\}^{n} \rightarrow\{0,1\}^{m}$. Let $L \subset[n]$ be a set of inputs, and $t, h \in[m]$. We say that $f$ is $(t, L, h)$-robust if for every set of outputs $T \subset[m]$ of size $t$ and every string $z \in\{0,1\}^{t}$ the following holds. Let $x \in\{0,1\}^{n}$ be a uniformly chosen string conditioned on the event $f(x)_{T}=z$, i.e., the outputs which are indexed by $T$ equal to $z$. Then the random variable $x_{L}=\left(x_{i}\right)_{i \in L}$ has min-entropy of $h$, namely, for every fixed $w \in\{0,1\}^{|L|}, \operatorname{Pr}\left[x_{L}=w\right] \leq 2^{-h}$. The function is $(t, \ell, h)$-robust if it is $(t, L, h)$-robust for every $\ell$-size input set $L$.

In the full version of this work, we prove that if $f_{G, P}$ is $k$-wise independent with respect to random graph, then it is also robust for shorter output length.

Lemma 6. Suppose that $P$ is a predicate for which $f_{G, P}:\{0,1\}^{n} \rightarrow\{0,1\}^{m}$ is $k$-wise independent, whp over the choice of a random $(m, n, d)$ graph $G$. Then, whp over the choice of a random $(m-r, n, d)$ graph $H$, the function $f_{H, P}$ : $\{0,1\}^{n} \rightarrow\{0,1\}^{m-r}$ is $(t, \ell, h)$-robust, where $h=\min \left(\ell, r \cdot(\ell / n)^{d} / 2, k-t\right)$.

In the case of linear stretch, $m=n+O(n)$, where $k$ is linear as well (Corollary 11), one can get $(t, \ell, h)$-robustness with linear parameters at the expense of linear decrease in the output length (e.g., $r=m / 2$ ). When the output is polynomial $m=n^{1+\varepsilon}$ (for $\left.\varepsilon<1 / 4\right)$, we get $(t, \ell, h)$-robustness for inverse-polynomial parameters, again at the expense of a linear decrease in the output length (e.g., $r=m / 2)$.

Robustness is especially useful if the actual number of preimages of $y=$ $f_{G, P}(x)$ is relatively small compared to $2^{h}$. In this case, an algorithm which attempts to guess $\ell$ bits of a preimage $x$ based on $t$ outputs is likely to be wrong 
(obtain a partial assignment that does not correspond to any preimage of $y$.) We show that in our setting of parameters (when the output length is large) most inputs have a small number of siblings under $f_{G, P}$ (where $G$ is random). The proof of the following lemma is given in the full version.

Lemma 7. Let $P$ be any nonconstant predicate. For every $\eta>0$ there exists a constant $M$ such that when $m>2^{M d} n \log n$,

$$
\underset{G, x}{\operatorname{Pr}}\left[\mid\left\{x^{\prime} \mid x^{\prime} \text { is a preimage of } f_{G, P}(x)\right\} \mid<M\right]>1-\eta \text {. }
$$

Myopic DPLL algorithms. We now show how the simple statistical properties proved in the above lemmas yield lower-bounds for DPLL algorithms who attack $f_{G, P}$. The high-level argument is similar to the one used in 316] and it is only sketched here. Consider the following myopic backtracking DPLL algorithm, whose input consists of $y=f_{G, P}(x)$ where $x$ is uniformly chosen. The algorithm is allowed to read the entire graph $G$, but it reads the values of $y$ in an incremental way. Specifically, in each iteration the algorithm adaptively chooses an input variable $x_{i}$ and asks to reveal $r$ new output bits of $y$. Then it guesses the value of $x_{i}$ based on its current state and on the output bits that were already revealed (including the ones that were revealed in previous iterations). If the algorithm reaches a contradiction, i.e., its partial assignment to $x$ is consistent with some output it backtracks.

Suppose that $f_{G, P}$ satisfies Lemmas 6 and 7 . Since $f_{G, P}$ is $k$-wise independent the algorithm does not backtrack in the first $k / r$ steps (as some patrial assignment is consistent with every value of $k$ outputs). Since $f$ is $(r \cdot \ell, \ell, h)$ robust and the number of siblings of a random $x$ is at most $M$ whp, the partial assignment chosen by the algorithm after $\ell<k$ steps is likely to be globally inconsistent (there are $2^{h}$ locally consistent assignments while there are only $M \ll 2^{h}$ globally consistent assignments). Hence, with all but negligible probability, the algorithm will err during the first $\ell$ steps, and therefore will backtrack at some point after more than $k$ steps. It can be shown (by standard lowerbound on resolution [10,2]) that, for a random graph, the backtracking phase takes super-polynomial time. (By plugging in the exact parameters the lowerbound is exponential $2^{\Omega(n)}$ when $m=O(n)$ or sub-exponential $\exp \left(n^{\delta}\right)$ when $m=n^{1+\varepsilon}$.)

\section{References}

1. Alekhnovich, M.: More on average case vs approximation complexity. In: FOCS, pp. 298-307. IEEE Computer Society (2003)

2. Alekhnovich, M., Ben-Sasson, E., Razborov, A.A., Wigderson, A.: Pseudorandom generators in propositional proof complexity. SIAM Journal of Computation 34(1), 67-88 (2004)

3. Alekhnovich, M., Hirsch, E.A., Itsykson, D.: Exponential lower bounds for the running time of DPLL algorithms on satisfiable formulas. J. Autom. Reasoning 35(1-3), $51-72(2005)$ 
4. Applebaum, B.: Pseudorandom generators with long stretch and low locality from random local one-way functions. Electronic Colloquium on Computational Complexity (ECCC) 18 (2011)

5. Applebaum, B., Barak, B., Wigderson, A.: Public-key cryptography from different assumptions. In: 42nd ACM Symposium on Theory of Computing (STOC 2010), pp. $171-180(2010)$

6. Applebaum, B., Ishai, Y., Kushilevitz, E.: Cryptography in $\mathrm{NC}^{0}$. SIAM Journal on Computing 36(4), 845-888 (2006)

7. Applebaum, B., Ishai, Y., Kushilevitz, E.: On pseudorandom generators with linear stretch in $\mathrm{NC}^{0}$. Journal of Computational Complexity 17(1), 38-69 (2008)

8. Applebaum, B., Ishai, Y., Kushilevitz, E.: Cryptography with constant input locality. Journal of Cryptology 22(4), 429-469 (2009)

9. Arora, S., Ge, R.: New Algorithms for Learning in Presence of Errors. In: Aceto, L., Henzinger, M., Sgall, J. (eds.) ICALP 2011, Part I. LNCS, vol. 6755, pp. 403-415. Springer, Heidelberg (2011)

10. Ben-Sasson, E., Wigderson, A.: Short proofs are narrow - resolution made simple. In: STOC, pp. 517-526 (1999)

11. Bogdanov, A., Papakonstantinou, P., Wan, A.: Pseudorandomness for read-once formulas. In: Proceedings of the 52nd Annual Symposium on Foundations of Computer Science (2011) (to appear)

12. Bogdanov, A., Qiao, Y.: On the Security of Goldreich's One-Way Function. In: Dinur, I., Jansen, K., Naor, J., Rolim, J. (eds.) APPROX and RANDOM 2009. LNCS, vol. 5687, pp. 392-405. Springer, Heidelberg (2009)

13. Bogdanov, A., Rosen, A.: Input Locality and Hardness Amplification. In: Ishai, Y. (ed.) TCC 2011. LNCS, vol. 6597, pp. 1-18. Springer, Heidelberg (2011)

14. Bogdanov, A., Viola, E.: Pseudorandom bits for polynomials. SIAM J. Comput. 39(6), 2464-2486 (2010)

15. Braverman, M.: Poly-logarithmic independence fools $\mathrm{AC}^{0}$ circuits. In: Annual IEEE Conference on Computational Complexity, pp. 3-8 (2009)

16. Cook, J., Etesami, O., Miller, R., Trevisan, L.: Goldreich's One-Way Function Candidate and Myopic Backtracking Algorithms. In: Reingold, O. (ed.) TCC 2009. LNCS, vol. 5444, pp. 521-538. Springer, Heidelberg (2009)

17. Cryan, M., Miltersen, P.B.: On Pseudorandom Generators in $\mathrm{NC}^{0}$. In: Sgall, J., Pultr, A., Kolman, P. (eds.) MFCS 2001. LNCS, vol. 2136, pp. 272-284. Springer, Heidelberg (2001)

18. Diakonikolas, I., Gopalan, P., Jaiswal, R., Servedio, R.A., Viola, E.: Bounded independence fools halfspaces. SIAM Journal of Computation 39(8), 3441-3462 (2010)

19. Diakonikolas, I., Kane, D.M., Nelson, J.: Bounded independence fools degree-2 threshold functions. In: FOCS, pp. 11-20 (2010)

20. Etesami, S.O.: Pseudorandomness against depth-2 circuits and analysis of goldreich's candidate one-way function. Technical Report EECS-2010-180, UC Berkeley (2010)

21. Goemans, M., Williamson, D.: Improved approximation algorithms for maximum cut and satisfiability problems using semidefinite programming. JACM: Journal of the ACM 42 (1995)

22. Goldreich, O.: Candidate one-way functions based on expander graphs. Electronic Colloquium on Computational Complexity (ECCC) 7(090) (2000)

23. Impagliazzo, R., Nisan, N., Wigderson, A.: Pseudorandomness for network algorithms. In: Proceedings of the 26th Annual ACM Symposium on Theory of Computing, pp. 356-364 (1994) 
24. Ishai, Y., Kushilevitz, E., Ostrovsky, R., Sahai, A.: Cryptography with constant computational overhead. In: Ladner, R.E., Dwork, C. (eds.) STOC, pp. 433-442. ACM (2008)

25. Itsykson, D.: Lower bound on average-case complexity of inversion of goldreich's function by drunken backtracking algorithms. In: Computer Science - Theory and Applications, 5th International Computer Science Symposium in Russia, pp. 204-215 (2010)

26. Miller, R.: Goldreich's one-way function candidate and drunken backtracking algorithms. Distinguished major thesis, University of Virginia (2009)

27. Mossel, E., Shpilka, A., Trevisan, L.: On $\epsilon$-biased generators in $\mathrm{NC}^{0}$. In: Proc. 44th FOCS, pp. 136-145 (2003)

28. Naor, J., Naor, M.: Small-bias probability spaces: Efficient constructions and applications. SIAM Journal on Computing 22(4), 838-856 (1993); Preliminary version in Proc. 22th STOC (1990)

29. Panjwani, S.K.: An experimental evaluation of goldreich's one-way function. Technical report, IIT, Bombay (2001)

30. Siegenthaler, T.: Correlation-immunity of nonlinear combining functions for cryptographic applications. IEEE Transactions on Information Theory 30(5), 776-778 (1984) 